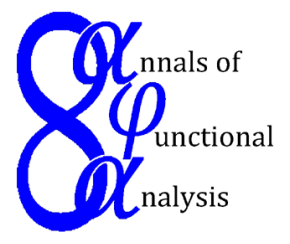

Ann. Funct. Anal. 7 (2016), no. 1, 102-117

http://dx.doi.org/10.1215/20088752-3429400

ISSN: 2008-8752 (electronic)

http://projecteuclid.org/afa

\title{
RECENT DEVELOPMENTS OF MATRIX VERSIONS OF THE ARITHMETIC-GEOMETRIC MEAN INEQUALITY
}

\author{
JUN ICHI FUJII, ${ }^{1}$ MASATOSHI FUJII, ${ }^{2}$ YUKI SEO, ${ }^{2}$ and HONGLIANG ZUO ${ }^{3}$ \\ Dedicated to Professor Anthony To-Ming Lau \\ Communicated by T. Yamazaki
}

\begin{abstract}
The main aim of this survey article is to present recent developments of matrix versions of the arithmetic-geometric mean inequality. Among others, we show improvements and generalizations of the arithmetic-geometric mean inequality for unitarily invariant norms via the Hadamard product, and for singular values via the operator monotone functions.
\end{abstract}

\section{IntRoduCtion}

A capital letter means an $n \times n$ matrix in the matrix algebra $\mathbb{M}_{n}$. For two Hermitian matrices $A, B$, the order relation $A \geq B$ means, by definition, that $A-B$ is positive semidefinite. Incidentally, $A \geq 0$ means that $A$ is positive semidefinite. Let us denote $A>0$ if $A$ is positive definite, that is, $A$ is positive semidefinite and invertible.

Matrix inequalities in this paper are of two kinds. One is based on the singular values. For a matrix $A \in \mathbb{M}_{n}$, the eigenvalues of $|A|:=\left(A^{*} A\right)^{1 / 2}$ are called the singular values of $A$ and are denoted by $s(A):=\left(s_{1}(A), s_{2}(A), \ldots, s_{n}(A)\right)$, which is arranged in decreasing order. For two matrices $A, B \in \mathbb{M}_{n}$, the order relation $s(A) \geq s(B)$ means that $s_{j}(A) \geq s_{j}(B)$ for $j=1, \ldots, n$. Notice the known fact that

$$
|A| \geq|B| \quad \Longrightarrow \quad s(A) \geq s(B)
$$

Copyright 2016 by the Tusi Mathematical Research Group.

Received Mar. 25, 2015; Accepted May 26, 2015.

2010 Mathematics Subject Classification. Primary 47A63; Secondary 15A18, 15A42, 47A30.

Keywords. arithmetic-geometric mean inequality, Heinz inequality, unitarily invariant norm, singular value, Hadamard product. 


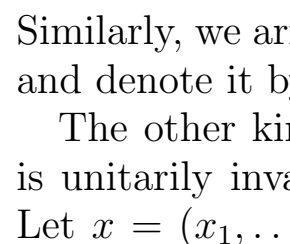

Ann. Funct. Anal. 7 (2016), no. 1, 102-117

http://dx.doi.org/10.1215/20088752-3429400

ISSN: 2008-8752 (electronic)

http://projecteuclid.org/afa

\title{
RECENT DEVELOPMENTS OF MATRIX VERSIONS OF THE ARITHMETIC-GEOMETRIC MEAN INEQUALITY
}

\author{
JUN ICHI FUJII, ${ }^{1}$ MASATOSHI FUJII, ${ }^{2}$ YUKI SEO, ${ }^{2}$ and HONGLIANG ZUO ${ }^{3}$ \\ Dedicated to Professor Anthony To-Ming Lau \\ Communicated by T. Yamazaki
}

\begin{abstract}
The main aim of this survey article is to present recent developments of matrix versions of the arithmetic-geometric mean inequality. Among others, we show improvements and generalizations of the arithmetic-geometric mean inequality for unitarily invariant norms via the Hadamard product, and for singular values via the operator monotone functions.
\end{abstract}

\section{IntRoduCtion}

A capital letter means an $n \times n$ matrix in the matrix algebra $\mathbb{M}_{n}$. For two Hermitian matrices $A, B$, the order relation $A \geq B$ means, by definition, that $A-B$ is positive semidefinite. Incidentally, $A \geq 0$ means that $A$ is positive semidefinite. Let us denote $A>0$ if $A$ is positive definite, that is, $A$ is positive semidefinite and invertible.

Matrix inequalities in this paper are of two kinds. One is based on the singular values. For a matrix $A \in \mathbb{M}_{n}$, the eigenvalues of $|A|:=\left(A^{*} A\right)^{1 / 2}$ are called the singular values of $A$ and are denoted by $s(A):=\left(s_{1}(A), s_{2}(A), \ldots, s_{n}(A)\right)$, which is arranged in decreasing order. For two matrices $A, B \in \mathbb{M}_{n}$, the order relation $s(A) \geq s(B)$ means that $s_{j}(A) \geq s_{j}(B)$ for $j=1, \ldots, n$. Notice the known fact that

$$
|A| \geq|B| \quad \Longrightarrow \quad s(A) \geq s(B)
$$

Copyright 2016 by the Tusi Mathematical Research Group.

Received Mar. 25, 2015; Accepted May 26, 2015.

2010 Mathematics Subject Classification. Primary 47A63; Secondary 15A18, 15A42, 47A30.

Keywords. arithmetic-geometric mean inequality, Heinz inequality, unitarily invariant norm, singular value, Hadamard product. 


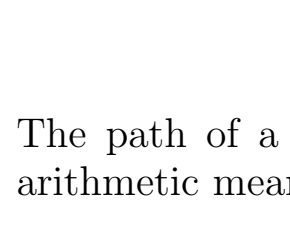

Ann. Funct. Anal. 7 (2016), no. 1, 102-117

http://dx.doi.org/10.1215/20088752-3429400

ISSN: 2008-8752 (electronic)

http://projecteuclid.org/afa

\title{
RECENT DEVELOPMENTS OF MATRIX VERSIONS OF THE ARITHMETIC-GEOMETRIC MEAN INEQUALITY
}

\author{
JUN ICHI FUJII, ${ }^{1}$ MASATOSHI FUJII, ${ }^{2}$ YUKI SEO, ${ }^{2}$ and HONGLIANG ZUO ${ }^{3}$ \\ Dedicated to Professor Anthony To-Ming Lau \\ Communicated by T. Yamazaki
}

\begin{abstract}
The main aim of this survey article is to present recent developments of matrix versions of the arithmetic-geometric mean inequality. Among others, we show improvements and generalizations of the arithmetic-geometric mean inequality for unitarily invariant norms via the Hadamard product, and for singular values via the operator monotone functions.
\end{abstract}

\section{IntRoduCtion}

A capital letter means an $n \times n$ matrix in the matrix algebra $\mathbb{M}_{n}$. For two Hermitian matrices $A, B$, the order relation $A \geq B$ means, by definition, that $A-B$ is positive semidefinite. Incidentally, $A \geq 0$ means that $A$ is positive semidefinite. Let us denote $A>0$ if $A$ is positive definite, that is, $A$ is positive semidefinite and invertible.

Matrix inequalities in this paper are of two kinds. One is based on the singular values. For a matrix $A \in \mathbb{M}_{n}$, the eigenvalues of $|A|:=\left(A^{*} A\right)^{1 / 2}$ are called the singular values of $A$ and are denoted by $s(A):=\left(s_{1}(A), s_{2}(A), \ldots, s_{n}(A)\right)$, which is arranged in decreasing order. For two matrices $A, B \in \mathbb{M}_{n}$, the order relation $s(A) \geq s(B)$ means that $s_{j}(A) \geq s_{j}(B)$ for $j=1, \ldots, n$. Notice the known fact that

$$
|A| \geq|B| \quad \Longrightarrow \quad s(A) \geq s(B)
$$

Copyright 2016 by the Tusi Mathematical Research Group.

Received Mar. 25, 2015; Accepted May 26, 2015.

2010 Mathematics Subject Classification. Primary 47A63; Secondary 15A18, 15A42, 47A30.

Keywords. arithmetic-geometric mean inequality, Heinz inequality, unitarily invariant norm, singular value, Hadamard product. 


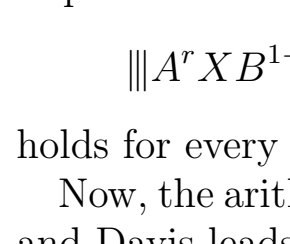

Ann. Funct. Anal. 7 (2016), no. 1, 102-117

http://dx.doi.org/10.1215/20088752-3429400

ISSN: 2008-8752 (electronic)

http://projecteuclid.org/afa

\title{
RECENT DEVELOPMENTS OF MATRIX VERSIONS OF THE ARITHMETIC-GEOMETRIC MEAN INEQUALITY
}

\author{
JUN ICHI FUJII, ${ }^{1}$ MASATOSHI FUJII, ${ }^{2}$ YUKI SEO, ${ }^{2}$ and HONGLIANG ZUO ${ }^{3}$ \\ Dedicated to Professor Anthony To-Ming Lau \\ Communicated by T. Yamazaki
}

\begin{abstract}
The main aim of this survey article is to present recent developments of matrix versions of the arithmetic-geometric mean inequality. Among others, we show improvements and generalizations of the arithmetic-geometric mean inequality for unitarily invariant norms via the Hadamard product, and for singular values via the operator monotone functions.
\end{abstract}

\section{IntRoduCtion}

A capital letter means an $n \times n$ matrix in the matrix algebra $\mathbb{M}_{n}$. For two Hermitian matrices $A, B$, the order relation $A \geq B$ means, by definition, that $A-B$ is positive semidefinite. Incidentally, $A \geq 0$ means that $A$ is positive semidefinite. Let us denote $A>0$ if $A$ is positive definite, that is, $A$ is positive semidefinite and invertible.

Matrix inequalities in this paper are of two kinds. One is based on the singular values. For a matrix $A \in \mathbb{M}_{n}$, the eigenvalues of $|A|:=\left(A^{*} A\right)^{1 / 2}$ are called the singular values of $A$ and are denoted by $s(A):=\left(s_{1}(A), s_{2}(A), \ldots, s_{n}(A)\right)$, which is arranged in decreasing order. For two matrices $A, B \in \mathbb{M}_{n}$, the order relation $s(A) \geq s(B)$ means that $s_{j}(A) \geq s_{j}(B)$ for $j=1, \ldots, n$. Notice the known fact that

$$
|A| \geq|B| \quad \Longrightarrow \quad s(A) \geq s(B)
$$

Copyright 2016 by the Tusi Mathematical Research Group.

Received Mar. 25, 2015; Accepted May 26, 2015.

2010 Mathematics Subject Classification. Primary 47A63; Secondary 15A18, 15A42, 47A30.

Keywords. arithmetic-geometric mean inequality, Heinz inequality, unitarily invariant norm, singular value, Hadamard product. 


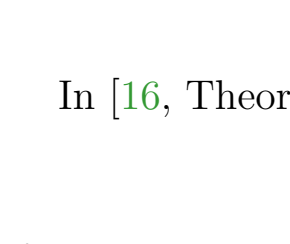

Ann. Funct. Anal. 7 (2016), no. 1, 102-117

http://dx.doi.org/10.1215/20088752-3429400

ISSN: 2008-8752 (electronic)

http://projecteuclid.org/afa

\title{
RECENT DEVELOPMENTS OF MATRIX VERSIONS OF THE ARITHMETIC-GEOMETRIC MEAN INEQUALITY
}

\author{
JUN ICHI FUJII, ${ }^{1}$ MASATOSHI FUJII, ${ }^{2}$ YUKI SEO, ${ }^{2}$ and HONGLIANG ZUO ${ }^{3}$ \\ Dedicated to Professor Anthony To-Ming Lau \\ Communicated by T. Yamazaki
}

\begin{abstract}
The main aim of this survey article is to present recent developments of matrix versions of the arithmetic-geometric mean inequality. Among others, we show improvements and generalizations of the arithmetic-geometric mean inequality for unitarily invariant norms via the Hadamard product, and for singular values via the operator monotone functions.
\end{abstract}

\section{IntRoduCtion}

A capital letter means an $n \times n$ matrix in the matrix algebra $\mathbb{M}_{n}$. For two Hermitian matrices $A, B$, the order relation $A \geq B$ means, by definition, that $A-B$ is positive semidefinite. Incidentally, $A \geq 0$ means that $A$ is positive semidefinite. Let us denote $A>0$ if $A$ is positive definite, that is, $A$ is positive semidefinite and invertible.

Matrix inequalities in this paper are of two kinds. One is based on the singular values. For a matrix $A \in \mathbb{M}_{n}$, the eigenvalues of $|A|:=\left(A^{*} A\right)^{1 / 2}$ are called the singular values of $A$ and are denoted by $s(A):=\left(s_{1}(A), s_{2}(A), \ldots, s_{n}(A)\right)$, which is arranged in decreasing order. For two matrices $A, B \in \mathbb{M}_{n}$, the order relation $s(A) \geq s(B)$ means that $s_{j}(A) \geq s_{j}(B)$ for $j=1, \ldots, n$. Notice the known fact that

$$
|A| \geq|B| \quad \Longrightarrow \quad s(A) \geq s(B)
$$

Copyright 2016 by the Tusi Mathematical Research Group.

Received Mar. 25, 2015; Accepted May 26, 2015.

2010 Mathematics Subject Classification. Primary 47A63; Secondary 15A18, 15A42, 47A30.

Keywords. arithmetic-geometric mean inequality, Heinz inequality, unitarily invariant norm, singular value, Hadamard product. 


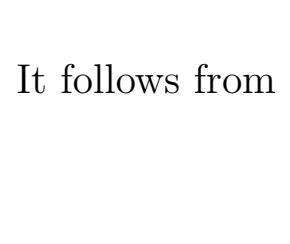

Ann. Funct. Anal. 7 (2016), no. 1, 102-117

http://dx.doi.org/10.1215/20088752-3429400

ISSN: 2008-8752 (electronic)

http://projecteuclid.org/afa

\title{
RECENT DEVELOPMENTS OF MATRIX VERSIONS OF THE ARITHMETIC-GEOMETRIC MEAN INEQUALITY
}

\author{
JUN ICHI FUJII, ${ }^{1}$ MASATOSHI FUJII, ${ }^{2}$ YUKI SEO, ${ }^{2}$ and HONGLIANG ZUO ${ }^{3}$ \\ Dedicated to Professor Anthony To-Ming Lau \\ Communicated by T. Yamazaki
}

\begin{abstract}
The main aim of this survey article is to present recent developments of matrix versions of the arithmetic-geometric mean inequality. Among others, we show improvements and generalizations of the arithmetic-geometric mean inequality for unitarily invariant norms via the Hadamard product, and for singular values via the operator monotone functions.
\end{abstract}

\section{IntRoduCtion}

A capital letter means an $n \times n$ matrix in the matrix algebra $\mathbb{M}_{n}$. For two Hermitian matrices $A, B$, the order relation $A \geq B$ means, by definition, that $A-B$ is positive semidefinite. Incidentally, $A \geq 0$ means that $A$ is positive semidefinite. Let us denote $A>0$ if $A$ is positive definite, that is, $A$ is positive semidefinite and invertible.

Matrix inequalities in this paper are of two kinds. One is based on the singular values. For a matrix $A \in \mathbb{M}_{n}$, the eigenvalues of $|A|:=\left(A^{*} A\right)^{1 / 2}$ are called the singular values of $A$ and are denoted by $s(A):=\left(s_{1}(A), s_{2}(A), \ldots, s_{n}(A)\right)$, which is arranged in decreasing order. For two matrices $A, B \in \mathbb{M}_{n}$, the order relation $s(A) \geq s(B)$ means that $s_{j}(A) \geq s_{j}(B)$ for $j=1, \ldots, n$. Notice the known fact that

$$
|A| \geq|B| \quad \Longrightarrow \quad s(A) \geq s(B)
$$

Copyright 2016 by the Tusi Mathematical Research Group.

Received Mar. 25, 2015; Accepted May 26, 2015.

2010 Mathematics Subject Classification. Primary 47A63; Secondary 15A18, 15A42, 47A30.

Keywords. arithmetic-geometric mean inequality, Heinz inequality, unitarily invariant norm, singular value, Hadamard product. 


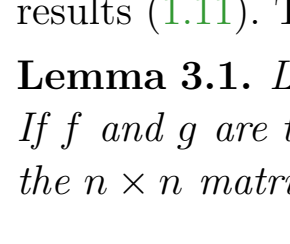

Ann. Funct. Anal. 7 (2016), no. 1, 102-117

http://dx.doi.org/10.1215/20088752-3429400

ISSN: 2008-8752 (electronic)

http://projecteuclid.org/afa

\title{
RECENT DEVELOPMENTS OF MATRIX VERSIONS OF THE ARITHMETIC-GEOMETRIC MEAN INEQUALITY
}

\author{
JUN ICHI FUJII, ${ }^{1}$ MASATOSHI FUJII, ${ }^{2}$ YUKI SEO, ${ }^{2}$ and HONGLIANG ZUO ${ }^{3}$ \\ Dedicated to Professor Anthony To-Ming Lau \\ Communicated by T. Yamazaki
}

\begin{abstract}
The main aim of this survey article is to present recent developments of matrix versions of the arithmetic-geometric mean inequality. Among others, we show improvements and generalizations of the arithmetic-geometric mean inequality for unitarily invariant norms via the Hadamard product, and for singular values via the operator monotone functions.
\end{abstract}

\section{IntRoduCtion}

A capital letter means an $n \times n$ matrix in the matrix algebra $\mathbb{M}_{n}$. For two Hermitian matrices $A, B$, the order relation $A \geq B$ means, by definition, that $A-B$ is positive semidefinite. Incidentally, $A \geq 0$ means that $A$ is positive semidefinite. Let us denote $A>0$ if $A$ is positive definite, that is, $A$ is positive semidefinite and invertible.

Matrix inequalities in this paper are of two kinds. One is based on the singular values. For a matrix $A \in \mathbb{M}_{n}$, the eigenvalues of $|A|:=\left(A^{*} A\right)^{1 / 2}$ are called the singular values of $A$ and are denoted by $s(A):=\left(s_{1}(A), s_{2}(A), \ldots, s_{n}(A)\right)$, which is arranged in decreasing order. For two matrices $A, B \in \mathbb{M}_{n}$, the order relation $s(A) \geq s(B)$ means that $s_{j}(A) \geq s_{j}(B)$ for $j=1, \ldots, n$. Notice the known fact that

$$
|A| \geq|B| \quad \Longrightarrow \quad s(A) \geq s(B)
$$

Copyright 2016 by the Tusi Mathematical Research Group.

Received Mar. 25, 2015; Accepted May 26, 2015.

2010 Mathematics Subject Classification. Primary 47A63; Secondary 15A18, 15A42, 47A30.

Keywords. arithmetic-geometric mean inequality, Heinz inequality, unitarily invariant norm, singular value, Hadamard product. 


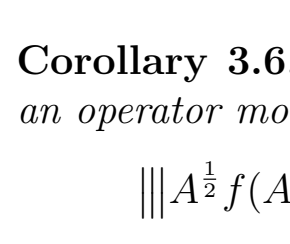

Ann. Funct. Anal. 7 (2016), no. 1, 102-117

http://dx.doi.org/10.1215/20088752-3429400

ISSN: 2008-8752 (electronic)

http://projecteuclid.org/afa

\title{
RECENT DEVELOPMENTS OF MATRIX VERSIONS OF THE ARITHMETIC-GEOMETRIC MEAN INEQUALITY
}

\author{
JUN ICHI FUJII, ${ }^{1}$ MASATOSHI FUJII, ${ }^{2}$ YUKI SEO, ${ }^{2}$ and HONGLIANG ZUO ${ }^{3}$ \\ Dedicated to Professor Anthony To-Ming Lau \\ Communicated by T. Yamazaki
}

\begin{abstract}
The main aim of this survey article is to present recent developments of matrix versions of the arithmetic-geometric mean inequality. Among others, we show improvements and generalizations of the arithmetic-geometric mean inequality for unitarily invariant norms via the Hadamard product, and for singular values via the operator monotone functions.
\end{abstract}

\section{IntRoduCtion}

A capital letter means an $n \times n$ matrix in the matrix algebra $\mathbb{M}_{n}$. For two Hermitian matrices $A, B$, the order relation $A \geq B$ means, by definition, that $A-B$ is positive semidefinite. Incidentally, $A \geq 0$ means that $A$ is positive semidefinite. Let us denote $A>0$ if $A$ is positive definite, that is, $A$ is positive semidefinite and invertible.

Matrix inequalities in this paper are of two kinds. One is based on the singular values. For a matrix $A \in \mathbb{M}_{n}$, the eigenvalues of $|A|:=\left(A^{*} A\right)^{1 / 2}$ are called the singular values of $A$ and are denoted by $s(A):=\left(s_{1}(A), s_{2}(A), \ldots, s_{n}(A)\right)$, which is arranged in decreasing order. For two matrices $A, B \in \mathbb{M}_{n}$, the order relation $s(A) \geq s(B)$ means that $s_{j}(A) \geq s_{j}(B)$ for $j=1, \ldots, n$. Notice the known fact that

$$
|A| \geq|B| \quad \Longrightarrow \quad s(A) \geq s(B)
$$

Copyright 2016 by the Tusi Mathematical Research Group.

Received Mar. 25, 2015; Accepted May 26, 2015.

2010 Mathematics Subject Classification. Primary 47A63; Secondary 15A18, 15A42, 47A30.

Keywords. arithmetic-geometric mean inequality, Heinz inequality, unitarily invariant norm, singular value, Hadamard product. 


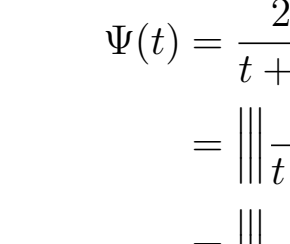

Ann. Funct. Anal. 7 (2016), no. 1, 102-117

http://dx.doi.org/10.1215/20088752-3429400

ISSN: 2008-8752 (electronic)

http://projecteuclid.org/afa

\title{
RECENT DEVELOPMENTS OF MATRIX VERSIONS OF THE ARITHMETIC-GEOMETRIC MEAN INEQUALITY
}

\author{
JUN ICHI FUJII, ${ }^{1}$ MASATOSHI FUJII, ${ }^{2}$ YUKI SEO, ${ }^{2}$ and HONGLIANG ZUO ${ }^{3}$ \\ Dedicated to Professor Anthony To-Ming Lau \\ Communicated by T. Yamazaki
}

\begin{abstract}
The main aim of this survey article is to present recent developments of matrix versions of the arithmetic-geometric mean inequality. Among others, we show improvements and generalizations of the arithmetic-geometric mean inequality for unitarily invariant norms via the Hadamard product, and for singular values via the operator monotone functions.
\end{abstract}

\section{IntRoduCtion}

A capital letter means an $n \times n$ matrix in the matrix algebra $\mathbb{M}_{n}$. For two Hermitian matrices $A, B$, the order relation $A \geq B$ means, by definition, that $A-B$ is positive semidefinite. Incidentally, $A \geq 0$ means that $A$ is positive semidefinite. Let us denote $A>0$ if $A$ is positive definite, that is, $A$ is positive semidefinite and invertible.

Matrix inequalities in this paper are of two kinds. One is based on the singular values. For a matrix $A \in \mathbb{M}_{n}$, the eigenvalues of $|A|:=\left(A^{*} A\right)^{1 / 2}$ are called the singular values of $A$ and are denoted by $s(A):=\left(s_{1}(A), s_{2}(A), \ldots, s_{n}(A)\right)$, which is arranged in decreasing order. For two matrices $A, B \in \mathbb{M}_{n}$, the order relation $s(A) \geq s(B)$ means that $s_{j}(A) \geq s_{j}(B)$ for $j=1, \ldots, n$. Notice the known fact that

$$
|A| \geq|B| \quad \Longrightarrow \quad s(A) \geq s(B)
$$

Copyright 2016 by the Tusi Mathematical Research Group.

Received Mar. 25, 2015; Accepted May 26, 2015.

2010 Mathematics Subject Classification. Primary 47A63; Secondary 15A18, 15A42, 47A30.

Keywords. arithmetic-geometric mean inequality, Heinz inequality, unitarily invariant norm, singular value, Hadamard product. 


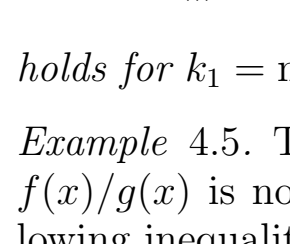

Ann. Funct. Anal. 7 (2016), no. 1, 102-117

http://dx.doi.org/10.1215/20088752-3429400

ISSN: 2008-8752 (electronic)

http://projecteuclid.org/afa

\title{
RECENT DEVELOPMENTS OF MATRIX VERSIONS OF THE ARITHMETIC-GEOMETRIC MEAN INEQUALITY
}

\author{
JUN ICHI FUJII, ${ }^{1}$ MASATOSHI FUJII, ${ }^{2}$ YUKI SEO, ${ }^{2}$ and HONGLIANG ZUO ${ }^{3}$ \\ Dedicated to Professor Anthony To-Ming Lau \\ Communicated by T. Yamazaki
}

\begin{abstract}
The main aim of this survey article is to present recent developments of matrix versions of the arithmetic-geometric mean inequality. Among others, we show improvements and generalizations of the arithmetic-geometric mean inequality for unitarily invariant norms via the Hadamard product, and for singular values via the operator monotone functions.
\end{abstract}

\section{IntRoduCtion}

A capital letter means an $n \times n$ matrix in the matrix algebra $\mathbb{M}_{n}$. For two Hermitian matrices $A, B$, the order relation $A \geq B$ means, by definition, that $A-B$ is positive semidefinite. Incidentally, $A \geq 0$ means that $A$ is positive semidefinite. Let us denote $A>0$ if $A$ is positive definite, that is, $A$ is positive semidefinite and invertible.

Matrix inequalities in this paper are of two kinds. One is based on the singular values. For a matrix $A \in \mathbb{M}_{n}$, the eigenvalues of $|A|:=\left(A^{*} A\right)^{1 / 2}$ are called the singular values of $A$ and are denoted by $s(A):=\left(s_{1}(A), s_{2}(A), \ldots, s_{n}(A)\right)$, which is arranged in decreasing order. For two matrices $A, B \in \mathbb{M}_{n}$, the order relation $s(A) \geq s(B)$ means that $s_{j}(A) \geq s_{j}(B)$ for $j=1, \ldots, n$. Notice the known fact that

$$
|A| \geq|B| \quad \Longrightarrow \quad s(A) \geq s(B)
$$

Copyright 2016 by the Tusi Mathematical Research Group.

Received Mar. 25, 2015; Accepted May 26, 2015.

2010 Mathematics Subject Classification. Primary 47A63; Secondary 15A18, 15A42, 47A30.

Keywords. arithmetic-geometric mean inequality, Heinz inequality, unitarily invariant norm, singular value, Hadamard product. 


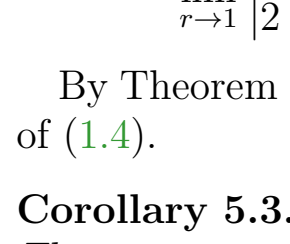

Ann. Funct. Anal. 7 (2016), no. 1, 102-117

http://dx.doi.org/10.1215/20088752-3429400

ISSN: 2008-8752 (electronic)

http://projecteuclid.org/afa

\title{
RECENT DEVELOPMENTS OF MATRIX VERSIONS OF THE ARITHMETIC-GEOMETRIC MEAN INEQUALITY
}

\author{
JUN ICHI FUJII, ${ }^{1}$ MASATOSHI FUJII, ${ }^{2}$ YUKI SEO, ${ }^{2}$ and HONGLIANG ZUO ${ }^{3}$ \\ Dedicated to Professor Anthony To-Ming Lau \\ Communicated by T. Yamazaki
}

\begin{abstract}
The main aim of this survey article is to present recent developments of matrix versions of the arithmetic-geometric mean inequality. Among others, we show improvements and generalizations of the arithmetic-geometric mean inequality for unitarily invariant norms via the Hadamard product, and for singular values via the operator monotone functions.
\end{abstract}

\section{IntRoduCtion}

A capital letter means an $n \times n$ matrix in the matrix algebra $\mathbb{M}_{n}$. For two Hermitian matrices $A, B$, the order relation $A \geq B$ means, by definition, that $A-B$ is positive semidefinite. Incidentally, $A \geq 0$ means that $A$ is positive semidefinite. Let us denote $A>0$ if $A$ is positive definite, that is, $A$ is positive semidefinite and invertible.

Matrix inequalities in this paper are of two kinds. One is based on the singular values. For a matrix $A \in \mathbb{M}_{n}$, the eigenvalues of $|A|:=\left(A^{*} A\right)^{1 / 2}$ are called the singular values of $A$ and are denoted by $s(A):=\left(s_{1}(A), s_{2}(A), \ldots, s_{n}(A)\right)$, which is arranged in decreasing order. For two matrices $A, B \in \mathbb{M}_{n}$, the order relation $s(A) \geq s(B)$ means that $s_{j}(A) \geq s_{j}(B)$ for $j=1, \ldots, n$. Notice the known fact that

$$
|A| \geq|B| \quad \Longrightarrow \quad s(A) \geq s(B)
$$

Copyright 2016 by the Tusi Mathematical Research Group.

Received Mar. 25, 2015; Accepted May 26, 2015.

2010 Mathematics Subject Classification. Primary 47A63; Secondary 15A18, 15A42, 47A30.

Keywords. arithmetic-geometric mean inequality, Heinz inequality, unitarily invariant norm, singular value, Hadamard product. 


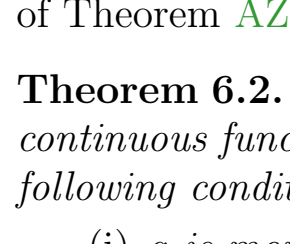

Ann. Funct. Anal. 7 (2016), no. 1, 102-117

http://dx.doi.org/10.1215/20088752-3429400

ISSN: 2008-8752 (electronic)

http://projecteuclid.org/afa

\title{
RECENT DEVELOPMENTS OF MATRIX VERSIONS OF THE ARITHMETIC-GEOMETRIC MEAN INEQUALITY
}

\author{
JUN ICHI FUJII, ${ }^{1}$ MASATOSHI FUJII, ${ }^{2}$ YUKI SEO, ${ }^{2}$ and HONGLIANG ZUO ${ }^{3}$ \\ Dedicated to Professor Anthony To-Ming Lau \\ Communicated by T. Yamazaki
}

\begin{abstract}
The main aim of this survey article is to present recent developments of matrix versions of the arithmetic-geometric mean inequality. Among others, we show improvements and generalizations of the arithmetic-geometric mean inequality for unitarily invariant norms via the Hadamard product, and for singular values via the operator monotone functions.
\end{abstract}

\section{IntRoduCtion}

A capital letter means an $n \times n$ matrix in the matrix algebra $\mathbb{M}_{n}$. For two Hermitian matrices $A, B$, the order relation $A \geq B$ means, by definition, that $A-B$ is positive semidefinite. Incidentally, $A \geq 0$ means that $A$ is positive semidefinite. Let us denote $A>0$ if $A$ is positive definite, that is, $A$ is positive semidefinite and invertible.

Matrix inequalities in this paper are of two kinds. One is based on the singular values. For a matrix $A \in \mathbb{M}_{n}$, the eigenvalues of $|A|:=\left(A^{*} A\right)^{1 / 2}$ are called the singular values of $A$ and are denoted by $s(A):=\left(s_{1}(A), s_{2}(A), \ldots, s_{n}(A)\right)$, which is arranged in decreasing order. For two matrices $A, B \in \mathbb{M}_{n}$, the order relation $s(A) \geq s(B)$ means that $s_{j}(A) \geq s_{j}(B)$ for $j=1, \ldots, n$. Notice the known fact that

$$
|A| \geq|B| \quad \Longrightarrow \quad s(A) \geq s(B)
$$

Copyright 2016 by the Tusi Mathematical Research Group.

Received Mar. 25, 2015; Accepted May 26, 2015.

2010 Mathematics Subject Classification. Primary 47A63; Secondary 15A18, 15A42, 47A30.

Keywords. arithmetic-geometric mean inequality, Heinz inequality, unitarily invariant norm, singular value, Hadamard product. 


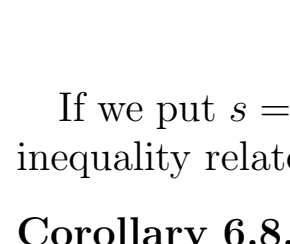

Ann. Funct. Anal. 7 (2016), no. 1, 102-117

http://dx.doi.org/10.1215/20088752-3429400

ISSN: 2008-8752 (electronic)

http://projecteuclid.org/afa

\title{
RECENT DEVELOPMENTS OF MATRIX VERSIONS OF THE ARITHMETIC-GEOMETRIC MEAN INEQUALITY
}

\author{
JUN ICHI FUJII, ${ }^{1}$ MASATOSHI FUJII, ${ }^{2}$ YUKI SEO, ${ }^{2}$ and HONGLIANG ZUO ${ }^{3}$ \\ Dedicated to Professor Anthony To-Ming Lau \\ Communicated by T. Yamazaki
}

\begin{abstract}
The main aim of this survey article is to present recent developments of matrix versions of the arithmetic-geometric mean inequality. Among others, we show improvements and generalizations of the arithmetic-geometric mean inequality for unitarily invariant norms via the Hadamard product, and for singular values via the operator monotone functions.
\end{abstract}

\section{IntRoduCtion}

A capital letter means an $n \times n$ matrix in the matrix algebra $\mathbb{M}_{n}$. For two Hermitian matrices $A, B$, the order relation $A \geq B$ means, by definition, that $A-B$ is positive semidefinite. Incidentally, $A \geq 0$ means that $A$ is positive semidefinite. Let us denote $A>0$ if $A$ is positive definite, that is, $A$ is positive semidefinite and invertible.

Matrix inequalities in this paper are of two kinds. One is based on the singular values. For a matrix $A \in \mathbb{M}_{n}$, the eigenvalues of $|A|:=\left(A^{*} A\right)^{1 / 2}$ are called the singular values of $A$ and are denoted by $s(A):=\left(s_{1}(A), s_{2}(A), \ldots, s_{n}(A)\right)$, which is arranged in decreasing order. For two matrices $A, B \in \mathbb{M}_{n}$, the order relation $s(A) \geq s(B)$ means that $s_{j}(A) \geq s_{j}(B)$ for $j=1, \ldots, n$. Notice the known fact that

$$
|A| \geq|B| \quad \Longrightarrow \quad s(A) \geq s(B)
$$

Copyright 2016 by the Tusi Mathematical Research Group.

Received Mar. 25, 2015; Accepted May 26, 2015.

2010 Mathematics Subject Classification. Primary 47A63; Secondary 15A18, 15A42, 47A30.

Keywords. arithmetic-geometric mean inequality, Heinz inequality, unitarily invariant norm, singular value, Hadamard product. 


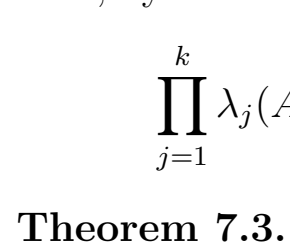

Ann. Funct. Anal. 7 (2016), no. 1, 102-117

http://dx.doi.org/10.1215/20088752-3429400

ISSN: 2008-8752 (electronic)

http://projecteuclid.org/afa

\title{
RECENT DEVELOPMENTS OF MATRIX VERSIONS OF THE ARITHMETIC-GEOMETRIC MEAN INEQUALITY
}

\author{
JUN ICHI FUJII, ${ }^{1}$ MASATOSHI FUJII, ${ }^{2}$ YUKI SEO, ${ }^{2}$ and HONGLIANG ZUO ${ }^{3}$ \\ Dedicated to Professor Anthony To-Ming Lau \\ Communicated by T. Yamazaki
}

\begin{abstract}
The main aim of this survey article is to present recent developments of matrix versions of the arithmetic-geometric mean inequality. Among others, we show improvements and generalizations of the arithmetic-geometric mean inequality for unitarily invariant norms via the Hadamard product, and for singular values via the operator monotone functions.
\end{abstract}

\section{IntRoduCtion}

A capital letter means an $n \times n$ matrix in the matrix algebra $\mathbb{M}_{n}$. For two Hermitian matrices $A, B$, the order relation $A \geq B$ means, by definition, that $A-B$ is positive semidefinite. Incidentally, $A \geq 0$ means that $A$ is positive semidefinite. Let us denote $A>0$ if $A$ is positive definite, that is, $A$ is positive semidefinite and invertible.

Matrix inequalities in this paper are of two kinds. One is based on the singular values. For a matrix $A \in \mathbb{M}_{n}$, the eigenvalues of $|A|:=\left(A^{*} A\right)^{1 / 2}$ are called the singular values of $A$ and are denoted by $s(A):=\left(s_{1}(A), s_{2}(A), \ldots, s_{n}(A)\right)$, which is arranged in decreasing order. For two matrices $A, B \in \mathbb{M}_{n}$, the order relation $s(A) \geq s(B)$ means that $s_{j}(A) \geq s_{j}(B)$ for $j=1, \ldots, n$. Notice the known fact that

$$
|A| \geq|B| \quad \Longrightarrow \quad s(A) \geq s(B)
$$

Copyright 2016 by the Tusi Mathematical Research Group.

Received Mar. 25, 2015; Accepted May 26, 2015.

2010 Mathematics Subject Classification. Primary 47A63; Secondary 15A18, 15A42, 47A30.

Keywords. arithmetic-geometric mean inequality, Heinz inequality, unitarily invariant norm, singular value, Hadamard product. 


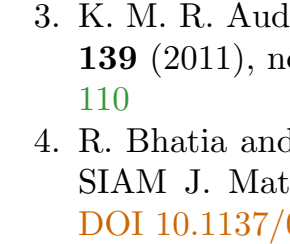

Ann. Funct. Anal. 7 (2016), no. 1, 102-117

http://dx.doi.org/10.1215/20088752-3429400

ISSN: 2008-8752 (electronic)

http://projecteuclid.org/afa

\title{
RECENT DEVELOPMENTS OF MATRIX VERSIONS OF THE ARITHMETIC-GEOMETRIC MEAN INEQUALITY
}

\author{
JUN ICHI FUJII, ${ }^{1}$ MASATOSHI FUJII, ${ }^{2}$ YUKI SEO, ${ }^{2}$ and HONGLIANG ZUO ${ }^{3}$ \\ Dedicated to Professor Anthony To-Ming Lau \\ Communicated by T. Yamazaki
}

\begin{abstract}
The main aim of this survey article is to present recent developments of matrix versions of the arithmetic-geometric mean inequality. Among others, we show improvements and generalizations of the arithmetic-geometric mean inequality for unitarily invariant norms via the Hadamard product, and for singular values via the operator monotone functions.
\end{abstract}

\section{IntRoduCtion}

A capital letter means an $n \times n$ matrix in the matrix algebra $\mathbb{M}_{n}$. For two Hermitian matrices $A, B$, the order relation $A \geq B$ means, by definition, that $A-B$ is positive semidefinite. Incidentally, $A \geq 0$ means that $A$ is positive semidefinite. Let us denote $A>0$ if $A$ is positive definite, that is, $A$ is positive semidefinite and invertible.

Matrix inequalities in this paper are of two kinds. One is based on the singular values. For a matrix $A \in \mathbb{M}_{n}$, the eigenvalues of $|A|:=\left(A^{*} A\right)^{1 / 2}$ are called the singular values of $A$ and are denoted by $s(A):=\left(s_{1}(A), s_{2}(A), \ldots, s_{n}(A)\right)$, which is arranged in decreasing order. For two matrices $A, B \in \mathbb{M}_{n}$, the order relation $s(A) \geq s(B)$ means that $s_{j}(A) \geq s_{j}(B)$ for $j=1, \ldots, n$. Notice the known fact that

$$
|A| \geq|B| \quad \Longrightarrow \quad s(A) \geq s(B)
$$

Copyright 2016 by the Tusi Mathematical Research Group.

Received Mar. 25, 2015; Accepted May 26, 2015.

2010 Mathematics Subject Classification. Primary 47A63; Secondary 15A18, 15A42, 47A30.

Keywords. arithmetic-geometric mean inequality, Heinz inequality, unitarily invariant norm, singular value, Hadamard product. 


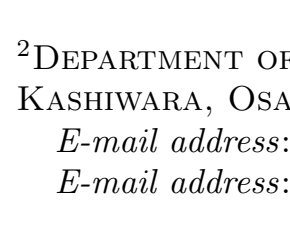

Ann. Funct. Anal. 7 (2016), no. 1, 102-117

http://dx.doi.org/10.1215/20088752-3429400

ISSN: 2008-8752 (electronic)

http://projecteuclid.org/afa

\title{
RECENT DEVELOPMENTS OF MATRIX VERSIONS OF THE ARITHMETIC-GEOMETRIC MEAN INEQUALITY
}

\author{
JUN ICHI FUJII, ${ }^{1}$ MASATOSHI FUJII, ${ }^{2}$ YUKI SEO, ${ }^{2}$ and HONGLIANG ZUO ${ }^{3}$ \\ Dedicated to Professor Anthony To-Ming Lau \\ Communicated by T. Yamazaki
}

\begin{abstract}
The main aim of this survey article is to present recent developments of matrix versions of the arithmetic-geometric mean inequality. Among others, we show improvements and generalizations of the arithmetic-geometric mean inequality for unitarily invariant norms via the Hadamard product, and for singular values via the operator monotone functions.
\end{abstract}

\section{IntRoduCtion}

A capital letter means an $n \times n$ matrix in the matrix algebra $\mathbb{M}_{n}$. For two Hermitian matrices $A, B$, the order relation $A \geq B$ means, by definition, that $A-B$ is positive semidefinite. Incidentally, $A \geq 0$ means that $A$ is positive semidefinite. Let us denote $A>0$ if $A$ is positive definite, that is, $A$ is positive semidefinite and invertible.

Matrix inequalities in this paper are of two kinds. One is based on the singular values. For a matrix $A \in \mathbb{M}_{n}$, the eigenvalues of $|A|:=\left(A^{*} A\right)^{1 / 2}$ are called the singular values of $A$ and are denoted by $s(A):=\left(s_{1}(A), s_{2}(A), \ldots, s_{n}(A)\right)$, which is arranged in decreasing order. For two matrices $A, B \in \mathbb{M}_{n}$, the order relation $s(A) \geq s(B)$ means that $s_{j}(A) \geq s_{j}(B)$ for $j=1, \ldots, n$. Notice the known fact that

$$
|A| \geq|B| \quad \Longrightarrow \quad s(A) \geq s(B)
$$

Copyright 2016 by the Tusi Mathematical Research Group.

Received Mar. 25, 2015; Accepted May 26, 2015.

2010 Mathematics Subject Classification. Primary 47A63; Secondary 15A18, 15A42, 47A30.

Keywords. arithmetic-geometric mean inequality, Heinz inequality, unitarily invariant norm, singular value, Hadamard product. 